\title{
MICROSTRUCTURAL ASPECTS OF FATIGUE CRACK INITIATION AND SHORT CRACK GROWTH IN RENÉ 88DT
}

\author{
Zafir Alam ${ }^{1, *}$, David Eastman ${ }^{1}$, George Weber $^{1}$, Somnath Ghosh ${ }^{1,2}$, Kevin Hemker ${ }^{1}$ \\ ${ }^{1}$ Department of Mechanical Engineering, Johns Hopkins University, Baltimore, MD, 21210, USA \\ ${ }^{2}$ Department of Civil Engineering, Johns Hopkins University, Baltimore, MD, 21210, USA
}

Keywords: Micro-bending fatigue, René 88DT superalloy, ICME

\begin{abstract}
The effect of local microstructure on fatigue crack initiation and propagation in polycrystalline René $88 \mathrm{DT}^{*}$ superalloy specimens has been examined at room temperature. Miniaturized samples were fatigue tested in reverse cycle bending in a novel resonance micro-bending fatigue setup. Concomitant analyses of the surface microstructure with orientation mapping, scanning electron microscopy, and confocal microscopy facilitated direct experimental measurements of the specific microstructural locations associated with: slip and slip band formation, microcrack initiation, and short-crack propagation. The underlying mechanisms were observed to be: slip on $\{111\}$ planes with the highest resolved shear stress, subsequent initiation of microcracks preferentially along, but not at, twin boundaries in large grains oriented for high shear and experiencing elastic incompatibility, and successive propagation of cracks on $\{111\}$ planes with high resolved shear stresses in the neighboring grains. Analyses of many short non-propagating cracks revealed crack arrest at high-angle grain boundaries.
\end{abstract}

\section{Introduction}

Components operating in gas turbine engines experience complex mechanical as well as thermal stress cycles. Fatigue is inevitable and a prominent mode of material failure in gas turbine engines [1-3]. The design of materials with fatigue resistant microstructures for enhanced durability of components and attendant engine performance forms a key objective of materials development which lies within the scope of the Materials Genome Initiative (MGI). In recent years, the development of fatigue life prediction models has gained impetus under the purview of Integrated Computational Materials Engineering (ICME) [4,5]. The MGI and ICME approaches require a thorough understanding of the response of local material microstructure to external loading. Insights on how local microstructure (grain size, orientation and neighborhood) influences local plasticity and subsequent crack formation and propagation, derived from experiments at salient length scales, are vital for the development and benchmarking of physics-based fatigue life prediction models.

In the present study, the effect of local microstructure on fatigue crack initiation and propagation in polycrystalline René 88DT has been examined at room temperature. Miniaturized samples with gage dimensions of $500 \mu \mathrm{m}$ in length, $500 \mu \mathrm{m}$ in width and 400 $\mu \mathrm{m}$ in thickness were fatigue tested in a resonance micro-bending fatigue setup that produced maximum stress at the surfaces. The surface microstructure of the gage was examined using orientation mapping, scanning electron microscopy (SEM), and confocal microscopy after intermittent cycles of testing. The specific microstructural locations associated with fatigue damage, i.e. slip

* René is a trademark of General Electric initiation, slip band formation, crack initiation, and crack propagation have been precisely identified.

\section{Experimental Details}

The material used was the polycrystalline Ni-based superalloy, René 88 DT. The nominal composition of the alloy is (in wt.\%) Ni-16Cr-13Co-4Mo-4W-3.7Ti-2.1Al-0.7Nb-0.03Zr-0.03C$0.015 \mathrm{~B}[6]$. The alloy was processed via a powder metallurgy route. Samples for experimental work were obtained from a disk forging as a block having dimensions $100 \mathrm{~mm}$ x $100 \mathrm{~mm}$ x 50 $\mathrm{mm}$. The forging received a solution heat treatment of $1 \mathrm{~h}$ at $1150^{\circ} \mathrm{C}$, followed by a delayed oil quench, and aged at $760^{\circ} \mathrm{C}$ for $8 \mathrm{~h}$. Sheets, $0.5 \mathrm{~mm}$ in thickness, were machined from the block by wire electrical discharge machining (EDM). Flat micro-bending fatigue samples (Figure 1(a)), with a gage section of $0.5 \mathrm{~mm}$ in length and $0.5 \mathrm{~mm}$ in width, were fabricated from the sheets by EDM. The sample was polished sequentially on 600, 800 and 1200 grit $\mathrm{SiC}$ papers. The gage section of the specimen was electro-polished in a Struers Lectropol-5 machine using an electrolyte mixture of (in vol.\%) 70-ethanol, 12-ethylene glycol monobutyl ether, 8-perchloric acid and 10-distilled water.

The resonance micro-bending fatigue tester, used in the present study, is identical to the one reported by Straub et al. [7]. Two linear actuators oscillate the sample while a laser position sensitive detector tracks the sample displacement (Figure. 1(b)). The samples were fatigue tested in pure bending mode at $20^{\circ} \mathrm{C}$. The initial resonance frequency (RF) of the samples was within the range of $390-400 \mathrm{~Hz}$ and the corresponding sample tip displacement was between 10-11 $\mu \mathrm{m}$. Accelerated fatigue testing was carried out by increasing the displacement to $13 \mu \mathrm{m}$. The stress and strain distribution in the sample was determined using 3-D elastic finite element simulations consisting of modal and dynamic analyses. In general, the test configuration was modeled using a commercial finite element code, Abaqus, with a 42,000 element mesh, consisting of 20-node quadratic brick elements. The modal study provided a computationally efficient method to determine the stress distributions and theoretical resonance frequency while the dynamic simulations were used as a method of confirmation for these results. From the geometry and boundary conditions, the natural frequencies of the structure were extracted through an eigenfrequency analysis. Dynamic simulations of the sample with the appropriate experimental boundary conditions were performed at the frequency of the first bending mode. The stress and strain field was assessed for a sample tip displacement corresponding to that of experiments, i.e. for $\delta_{\mathrm{A}}=13 \mu \mathrm{m}$. The sample gage surface experienced the maximum tensile stress with a magnitude of $80 \mathrm{MPa}$ while the shear stress was zero. The stress state of the gage surface alternated between tensile and compressive, i.e. fully reversible $(\mathrm{R}=-1)$, throughout the tests. 


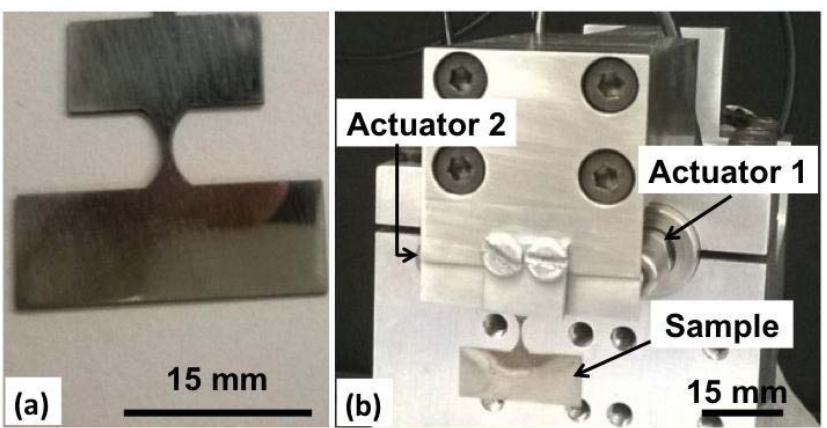

Figure 1. Photographs showing (a) the micro-bending fatigue sample and (b) the resonance micro-bending fatigue tester.

Damage initiation in the sample gage section causes a decrease in $\mathrm{RF}$, and the tests were carried out until various levels of decrease in resonance frequency $(\triangle \mathrm{RF})$ ranging between $0.25-1.0 \%$ were produced. After each interval of fatigue testing, the surface microstructure of the sample gage was examined using laser confocal profilometry, SEM and electron back scattered diffraction (EBSD) techniques in a Tescan SEM-EBSD machine operated at $20 \mathrm{kV}$. Analyses of EBSD data were carried out using the commercially available TSL OIM ${ }^{*}$ software and the crystallographic aspects of slip within the grains and subsequent crack propagation across grains ascertained. The microstructural evolution is reported with respect to cumulative changes in $\triangle \mathrm{RF}$ and number of fatigue cycles $(\mathrm{N})$.

\section{Results}

The initial microstructure was constituted of randomly oriented equiaxed grains containing twins (Figure 2). The fraction of twin boundaries, measured with respect to the overall boundaries, was 0.51 . The twin boundaries were coherent, parallel to $\{111\}$, i.e. of the $\Sigma 3$ type, and are indicated by red contours. The grain size varied in the range between 5-40 $\mu \mathrm{m}$ with an average value of $\sim 20$ $\mu \mathrm{m}$. In this orientation imaging microscopy (OIM) map, the highangle grain boundaries (HAGBs) denoted in black contours demarcate neighboring grains having misorientation greater than $15^{\circ}$. The blue contours indicate low angle-grain boundaries (LAGBs). The fraction of LAGBs was very low (0.02) as compared to the HAGBs (0.47). Further details on the microstructure of the René 88DT superalloy have been reported by Wlodek et al. [6] and Miao et al. [8,9].

\section{$\underline{\text { Slip and Microcrack Initiation }}$}

After fatigue testing for 150,000 cycles, corresponding to $\Delta \mathrm{RF}$ of $0.25 \%$, slip was observed in a select few grains, as evident from the fine slip bands indicated in Figure 3(a). The corresponding OIM image is shown in Figure 3(b). Within each grain, the slip bands were parallel to each other and confined within the HAGBs. Figures 3(c) and 3(d) show the maps for the Schmid factor (SF) and elastic stiffness along the loading direction $\left(\mathrm{E}_{\mathrm{LD}}\right)$ of the sample. The slip bands were observed to initiate within grains and twins exhibiting a high Schmid factor (SF > 0.43), irrespective of their size.

The formation of a microcrack can also be seen in Figure 3(a). The microcrack was constituted of fine slip bands within which

${ }^{*}$ TSL OIM is a trademark of AMETEK Inc.

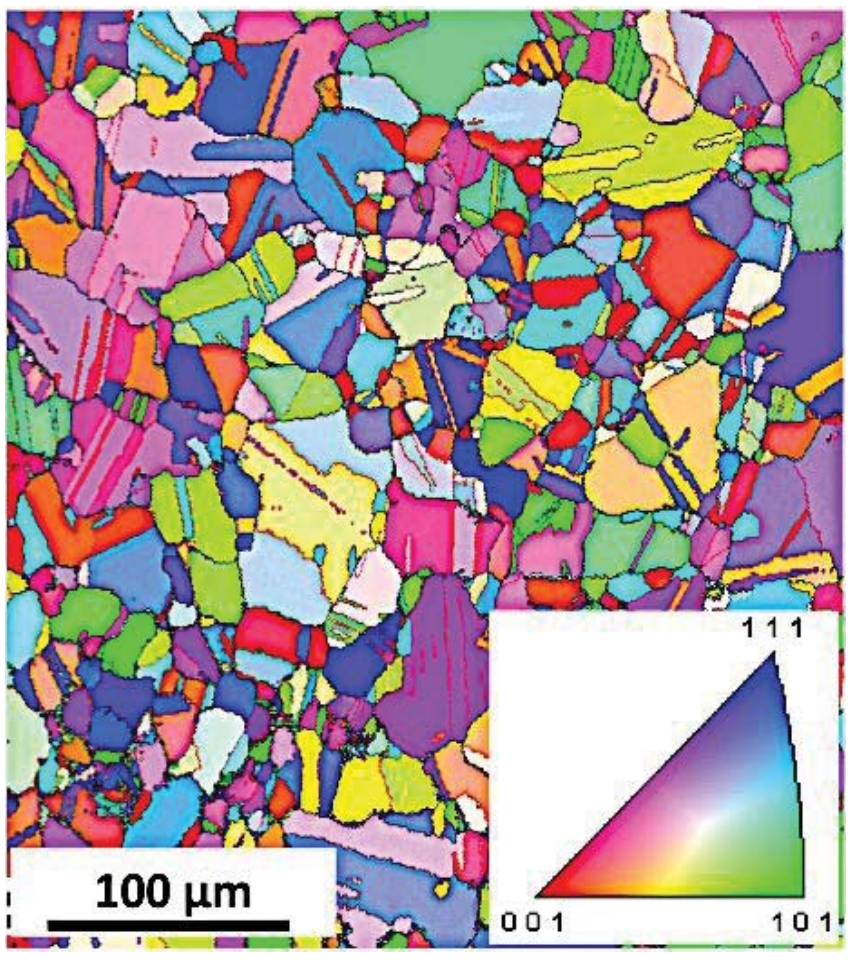

Figure 2. EBSD inverse pole figure of the microstructure of the René 88DT samples prior to fatigue testing. Please refer to the $\mathrm{PDF}$ of the manuscript on the Conference CD for the color image.

extrusions and intrusions had formed, as evident from the magnified image (inset in Figure 3(a)). A combined assessment with the OIM (Figure 3(b)) revealed that the microcrack had formed in the vicinity of a twin boundary. Closer inspection indicates that the microcrack formed parallel to the twin boundary, but not at the twin boundary, and was offset from the twin boundary (Figure 4(a)). Figure 3(c) indicates that the entire grain (G1) containing the twin (T1) was oriented for high shear $(\mathrm{SF}=0.46)$. Additionally, an elastic mismatch (difference in $\left.\mathrm{E}_{\mathrm{LD}}\right)$ was also observed across the twin boundary (Figure 3(d)). About 10 other microcracks were detected on the gage and, in each case, the microcracks formed in slip bands parallel to (but not at) twin boundaries. Furthermore, these microcracks had formed when the twin boundary experienced a high resolved shear stress and elastic mismatch. Lastly, such microcrack initiation was only observed in large grains that had a size comparable to or greater than the average grain size of $20 \mu \mathrm{m}$. The above observations are similar to that reported by Miao et al $[8,9]$.

In face centered cubic alloys slip occurs on $\{111\}$ planes with the highest resolved shear stress, so the orientation of the $\{111\}$ planes was highlighted in the OIM maps and the slip bands were found to lie parallel to specific $\{111\}$ planes for each grain. The Schmid factor was determined for all $\{111\}<110>$ slip systems in the grains that exhibited slip bands and subsequent microcrack formation and in all cases the slip bands and cracks occurred on planes with the highest Schmid factor. This is illustrated for the ensemble comprising the grain G1 and twin T1 (Figure 4(a)), the Schmid factor within G1 was the highest for (111)[1-10] (Figure 4(b)). The slip band within the twin T1 occurred by slip on (111)[10-1] since the Schmid factor was the highest for this slip system (Figure 4(c)). Simultaneously, the twin boundary plane 
was also (111) which indicated that the twin boundary was oriented for high shear (Figure 4(a)). A similar approach was adopted for the precise identification of slip characteristics associated with the other microcracks. In all cases, the slip bands had formed on $\{111\}$ planes experiencing the highest resolved shear stress and the twin boundaries, parallel to which the microcracks formed, were oriented for high shear. The angle between the trace of these twin boundaries and the loading axis was measured to be within $35-70^{\circ}$, which further corroborates that these critical twin boundaries were oriented for high shear [10].

By comparison, no microcracks initiated where the twin boundary plane was not the $\{111\}$ with the highest Schmid factor, even with differences in elastic stiffness across the twin boundary. In these instances, intersection of slip bands with the twin boundaries gave rise to a zig-zag slip trace as the slip band continued to propagate.
Similarly, no microcracking occurred along the slip bands that had formed within the grain interiors or the twins where the twin boundary was not oriented for high shear. The surface profile was measured across the various slip bands. The height of the slip bands which formed parallel to the twin boundaries was higher $(\sim 60 \mathrm{~nm})$ than that of the other slip bands $(\sim 20 \mathrm{~nm})$, suggesting greater slip offsets in the former.

The observations can be summarized as follows: (i) slip occurs on $\{111\}$ planes experiencing the highest resolved shear stress in the grains as well as the twins, and (ii) microcracks form parallel to (but not at) the twin boundaries in large grains oriented for a high resolved shear stress and where the twin creates an elastic mismatch.
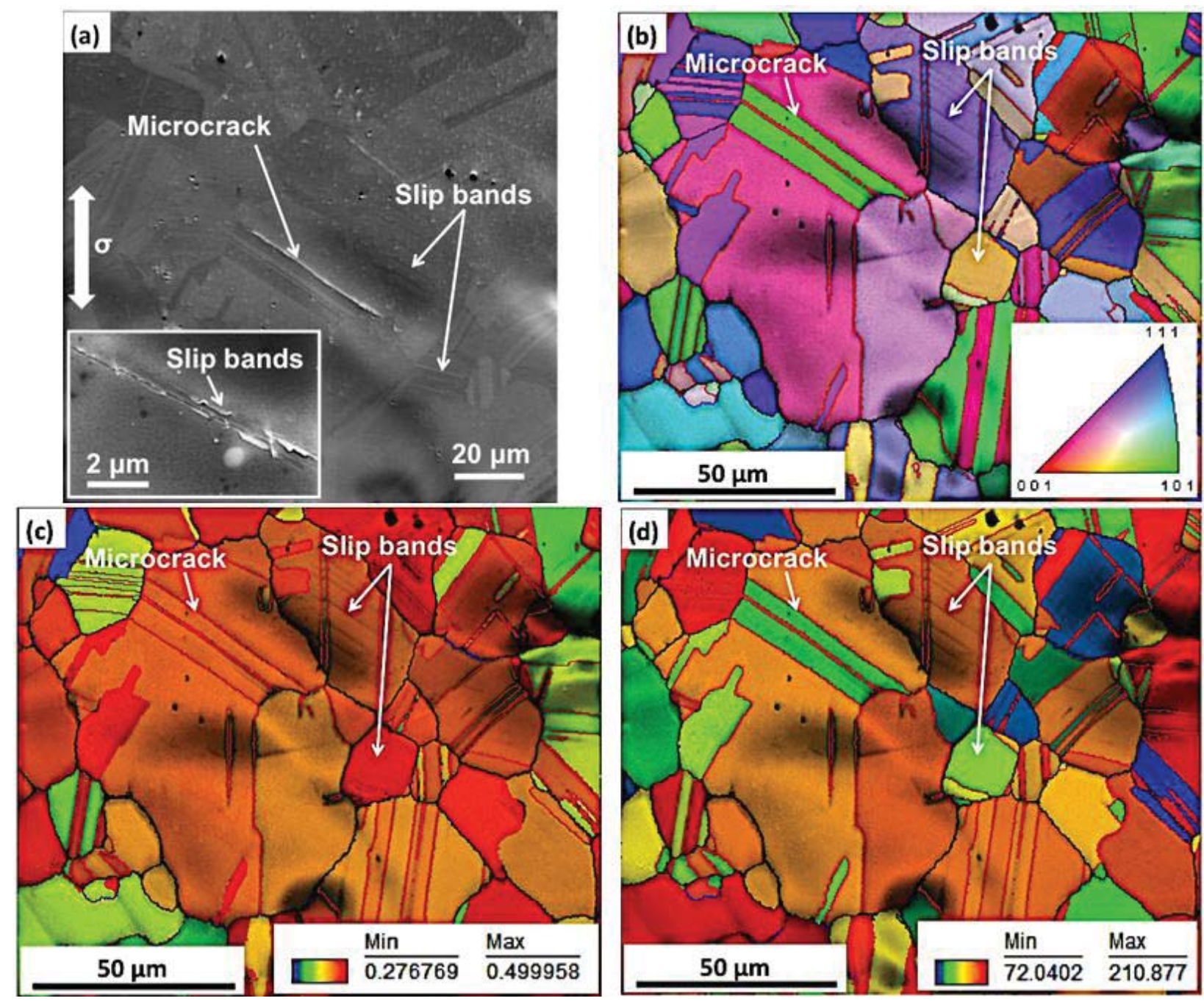

Figure 3. (a) Formation of slip bands and microcrack, (b) Orientation Imaging Microscopy (OIM) map, and (c,d) Schmid factor (SF) and modulus $\left(\mathrm{E}_{\mathrm{LD}}\right)$ maps. The loading direction $(\mathrm{LD})$ is vertical and denoted by $\sigma$. Please refer to the PDF of the manuscript on the Conference $\mathrm{CD}$ for the color images. 


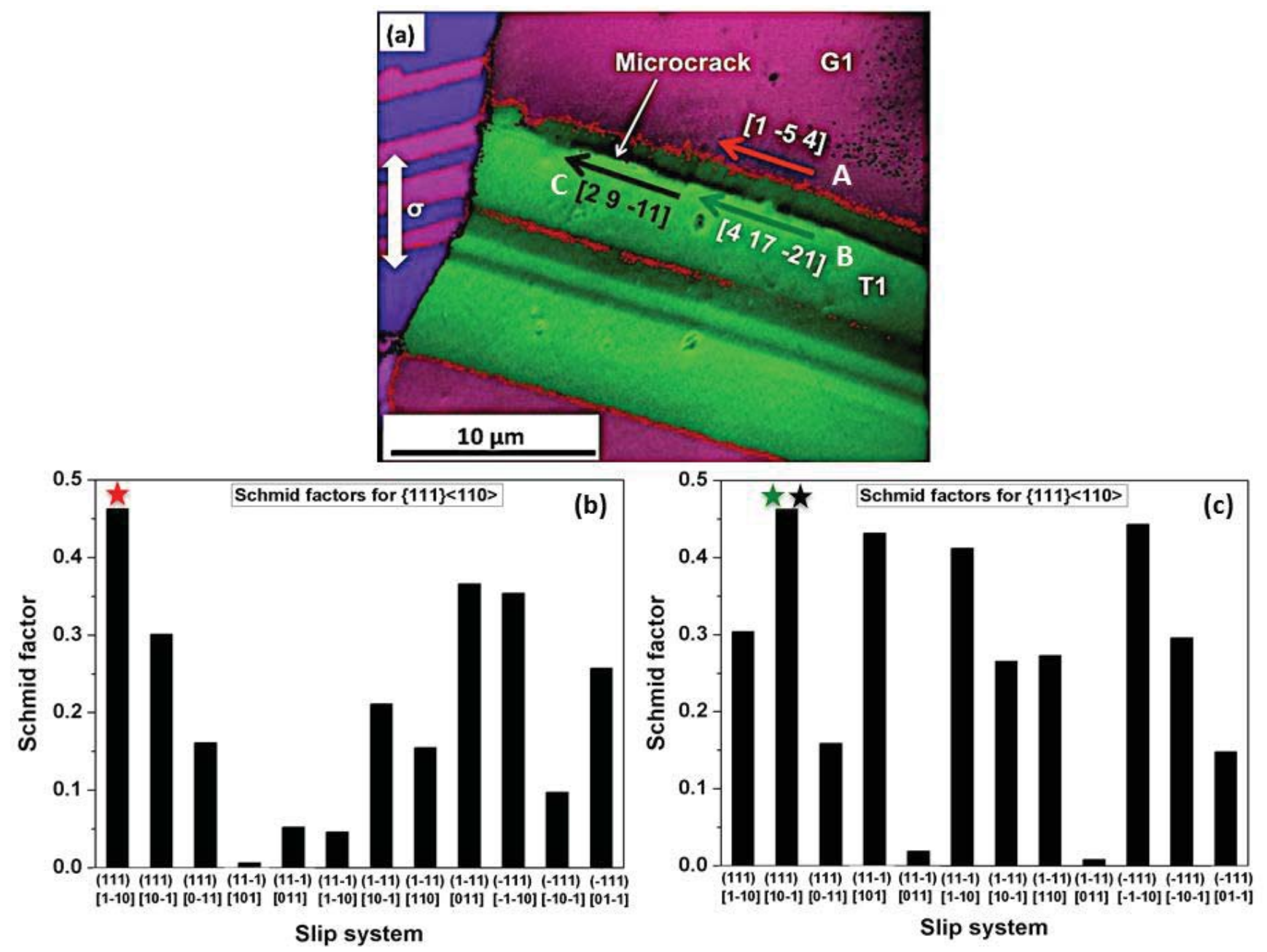

Figure 4. (a) OIM map showing that the microcrack is offset from the twin boundary. The red (A), green (B) and black (C) arrows denote the respective crystal direction along the twin boundary, microcrack and the microcrack/slip band within the twin. The Schmid factor (SF) plots for the various $\{111\}<110>$ slip systems in grain G1 and twin T1 are shown in figures $\mathrm{b}$ and $\mathrm{c}$. The star legends denote the highest SF for the $\{111\}$ planes corresponding to the crystal directions shown in (a). Please refer to the PDF of the manuscript on the Conference CD for the color images.

\section{Short-crack Growth}

After fatigue testing for 500,000 cycles $(\Delta \mathrm{RF}=0.5 \%)$, the microcracks were observed to have propagated into neighboring grains and extended across about five grains. A snapshot of the growth path for one such crack is given in Figure 5(a). The microcrack originated in grain $\mathrm{G} 1$, traversed across the HAGB between G1 and G2, grew successively into the neighboring grains G2, G3 and G4. It was interesting to note that the crack did not propagate along the stack of lamellar twin boundaries in G2. Rather, the crack propagated across the twins in G2, into the grain G3 and subsequently parallel to the twin boundary in the grain G4 before it was found to arrest at a HAGB in the top left corner of the micrograph.

In order to understand such crack propagation behavior, the variation in Schmid factor and $\mathrm{E}_{\mathrm{LD}}$ was analyzed for each grain. By virtue of its orientation, G2 experienced low shear stress but was elastically more rigid, i.e. exhibited higher value of elastic stiffness, when compared to that of G3 and G4, as shown in Figures 5(b) and 5(c). Furthermore, the elastic difference between twin and matrix was very low in G2 (Figure 5(b)). By contrast, the elastic stiffness $\left(E_{L D}\right)$ for $G 3$ was the lowest but there was appreciable elastic incompatibility between the twin and matrix in G4 (Figure 5(c)). The variation in Schmid factor for the various $\{111\}<110>$ slip systems in G2 is given in Figure 5(d). Based on the direction of the trace of the twin boundary (red arrow A in Figure 5(a)), the twin plane was determined to be (1-11), which had a maximum Schmid factor of 0.19 (Figure 5(d)). On the other hand, the direction of the trace corresponding to crack propagation (grey arrow B in Figure 5(a)) indicated the crack plane as (11-1), which had a much higher Schmid factor value of 0.37 in G2 (Figure 5(d)). These observations clearly indicate that the propagation of cracks in the neighboring grains occurred on the $\{111\}$ planes with the highest Schmid factor. The crack and along the twin boundary in G4, where both G3 and G4 experience 

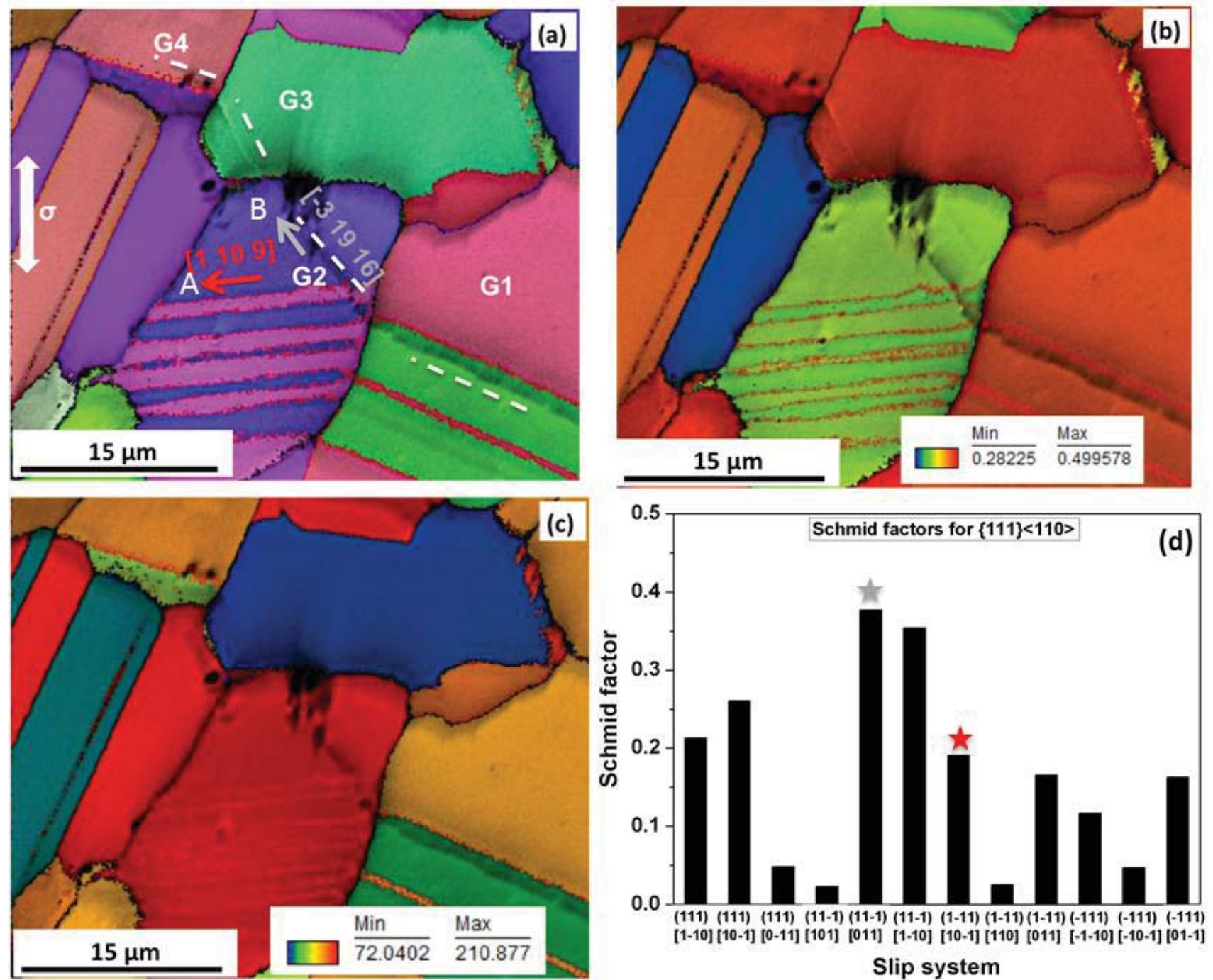

Figure 5. (a) OIM map showing the propagation of the microcrack across the twins in grain G2 and subsequently into grains G3 and G4, (b) Schmid factor map, and (c) $E_{\mathrm{LD}}$ map. The highest SF for the twin plane and the plane of crack propagation, indicated by arrows in (a), are shown by the corresponding star legends in (d). The loading direction is denoted by $\sigma$. The crack path is indicated by dashed lines in (a). Please refer to the PDF of the manuscript on the Conference CD for the color images.

high shear stress (Figure 4(d)). Similar observations of transgranular crack propagation across grains, along $\{111\}$ planes with high Schmid factor, were collected for many short cracks. The short cracks were observed to arrest at occasional HAGBs.

\section{$\underline{\text { Long-crack Formation }}$}

After fatigue testing for $1,000,000$ cycles $(\Delta \mathrm{RF}=1 \%)$, the formation of much longer cracks was observed. The crack path exhibited straight as well as zig-zag sections (Figure 6(a)). Slip trace analyses revealed that the growth of cracks occurred exclusively on $\{111\}$ planes and that for each grain (or twin) the cracks propagated along the $\{111\}$ plane with the highest resolved shear stress (Figure 6(b)). The zig-zag sections correspond to crack propagation on multiple $\{111\}<110>$ slip systems having similar Schmid factor while the straight sections denote the propagation of the crack along twin boundaries (Figures $6(a, b)$ ).
Coalescence of propagating cracks to form long continuous cracks was also observed.

\section{Discussion}

The mechanism of microcrack initiation can be described as follows. During the initial stages of fatigue, slip initiates on $\{111\}$ planes experiencing the highest Schmid factor $(\mathrm{SF}>0.43)$, irrespective of the grain size (Figure 3(a)). The slip bands, representing regions of intense strain localization, form and increase in width and depth with fatigue loading [11]. Each slip band is constituted of several thousands of atomic planes and the relative displacements amongst these planes results in the formation of extrusions and intrusions [11]. The surface offsets at the slip band/matrix interface as well as amongst extrusions/intrusions are thought to facilitate the initiation of microcracks by shear decohesion of the interface [11]. The observations made in the current study indicate that the grains and 
twins oriented for high resolved shear stress exhibit the formation of slip bands; however, microcracks only initiate in certain slip bands (Figure 3(a)). The grain size, orientation of the grains as well as the presence of twins, and orientation of the twin boundaries with respect to the slip band play crucial roles in microcracking. Four criteria should be concurrently satisfied for the initiation of microcracks: (i) the size of grains containing the twins should be greater than or equal to the average grain size, (ii) the grain containing the twin should be oriented to give a high resolved shear stress ( $\mathrm{SF}>0.43$ ), (iii) the twin boundary must be oriented for high Schmid factor, i.e. the slip band should form parallel to the twin boundaries, and (iv) there must be elastic incompatibility across the twin boundary. Similar microstructural constraints for initiation of fatigue microcracks in René 88DT superalloy have also been reported by Miao et al. [8,9] and Stinville et al. [12,13].

The orientation of slip bands with respect to the twin boundaries is an important factor in determining whether slip will be transmitted across the twin boundaries or microcracks initiate at the twin boundaries. When the twin boundaries are not oriented for maximum shear, the slip bands impinge with the twin boundaries, transmissal of slip occurs and no microcracks are formed. On the other hand, microcracks form only when the slip bands form parallel to the twin boundaries and do not impinge with them, i.e. when the twin boundaries are oriented for maximum shear (Figures 3 and 4). The microcracks do not form at the twin boundaries but are offset from the twin boundary, as observed in the present study (Figure 4(a)) as well as in previous studies $[8,9,12,13]$. Stinville et al., using high-resolution digital image correlation (DIC) for strain measurement, have reported that the local strain accumulation in the vicinity of twin boundaries oriented for maximum shear can be 8-10 times higher than that of the global strain [12]. On the other hand, the local strain accumulation remains low, about 0.2-0.9 times that of the global strain, for other orientations of the twin boundaries. They also report a three-fold escalation in the shear strains in the vicinity of twin boundaries oriented for high shear, which led to local stress concentration and microcracking [12]. The elastic incompatibility across these twin boundaries further assists in the formation of microcracks $[12,13]$. It is to be noted that the crack always forms in the elastically less rigid lattice near the twin boundary though the Schmid factor for the entire grain including the twin remains the same (Figures 3(d) and 4(a)). The elastic strain in the lattice oriented for low elastic stiffness would be greater than that of an elastically rigid lattice for a given stress. Therefore, the excess elastic strain is constrained within the elastically compliant matrix. It is known that the strains are not fully relieved even during fully reversible fatigue loading which results in strain accumulation [11]. The formation of microcracks near the twin boundaries can thus be ascribed to the localized accumulation of strain during the progressive fatigue loading cycles. This aspect can be correlated with the higher slip offsets associated with the slip bands that form near the twin boundaries than that of the slip bands away from the twin boundary, as mentioned earlier.
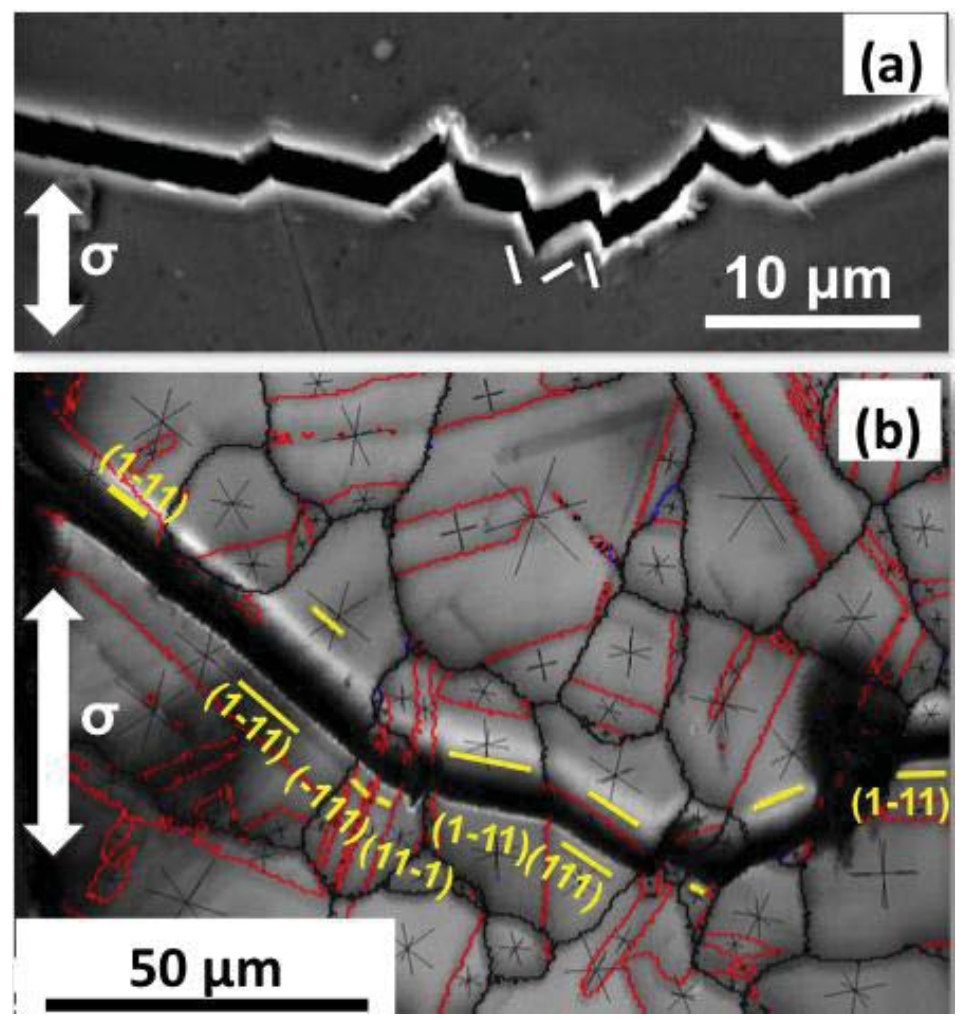

Figure 6. (a) Crack path showing straight and zig-zag sections (indicated by short white lines), and (b) image quality map showing crack propagation across high-angle grain boundaries and twin boundaries. The trace of various $\{111\}$ planes with high SF is superimposed in each grain and the plane of crack propagation is indicated in yellow. The loading direction is denoted by $\sigma$. Please refer to the PDF of the manuscript on the Conference $\mathrm{CD}$ for the color images. 
Slip was observed in small and large grains with high Schmid factor; but, the microcracks preferentially formed near twin boundaries in grains that were larger than the average grain size of $20 \mu \mathrm{m}$. The high shear stresses in the vicinity of twin boundaries oriented for maximum shear have been related to a high density of dislocations as well as shearing of the gamma prime precipitates $[12,14]$. Since the slip bands form parallel to and do not impinge with the twin boundary, the dislocations within the slip bands can glide parallel to the twin boundary when the twin plane happens to be the $\{111\}$ with the highest Schmid factor [14]. The dislocation glide is assisted by the stress concentration arising from elastic incompatibility across the twin boundary, which leads to strain localization [14]. Longer twins, contained in larger grains, are likely to provide larger glide lengths, assist in strain localization and promote microcracking. The number of gamma prime precipitates sheared along the glide plane can also enhance local stress concentration and affect microcracking. [12].

The observation that during short crack growth the cracks propagate along the $\{111\}$ plane with the highest resolved shear stress is counter intuitive and deserves further attention. It is well documented that dislocation motion is directly related to the critically resolved shear stress on the slip plane in the direction of the Burgers vector, but crack propagation is generally thought to occur on planes with high normal tensile stresses. The cracks observed in this study are primarily mode I cracks and their dependence on resolved shear stress is rather perplexing. The anomaly can, however, be explained based on the slip offsets associated with the slip bands. These slip offsets manifest as surface grooves along the slip band and increase in depth as well as width with increasing numbers of fatigue cycles. Therefore, once a microcrack nucleates its propagation is confined to within the surface groove that is formed along the slip band. The microcrack and the slip band both grow incrementally during the progressive cycles of fatigue loading. Thus, the propagation of the microcracks leads to the formation of short cracks, both of which propagate along $\{111\}$ planes, and ultimately into long cracks, which align themselves with the macroscopic stress state and lead to final failure.

\section{Summary}

In summary, the present paper describes the role of microstructure on fatigue crack initiation and propagation in a polycrystalline $\mathrm{Ni}$ base superalloy, René 88DT. It employed a unique experimental tool comprised of a resonance micro-bending fatigue setup and concomitant orientation imaging as well as confocal microscopy. Microcracks were observed to form along (but not at) the twin boundaries in large grains oriented with a high Schmid factor and where elastic incompatibility exists across the twin boundary. Moreover, the short crack growth of cracks in the neighboring grains was also observed to occur on $\{111\}$ planes oriented for high resolved shear stresses. The physics of fatigue crack initiation and propagation, ascertained in the present study, can serve as inputs for the development and validation of microstructure specific ICME fatigue models at the meso-scale.

\section{Acknowledgements}

This work has been supported through a grant No. FA9550-12-10445 to the Center of Excellence on Integrated Materials Modeling (CEIMM) at Johns Hopkins University awarded by the AFOSR/RSL Computational Mathematics Program, (Manager Dr.
A. Sayir) and AFRL/RX (Monitors Drs. C. Woodward and C. Przybyla). We also acknowledge A. Loghin, D. Konitzer, J. Williams and J. Marte of GE Global Research and J.C. Stinville and T. Pollock for providing René 88DT and useful discussions.

\section{References}

1. B.A. Cowles. "High Cycle Fatigue in Aircraft Gas Turbinesan Industry Perspective", International Journal of Fracture 80 (1996), 147-163.

2. M.R. Winstone, J.W. Brooks. "Advanced High Temperature Materials: Aeroengine Fatigue", Ciência e Technologia dos Materials, 20(1/2) (2008), 15-24.

3. C.B. Meher-Homji, G. Gabriles. "Gas Turbine Blade FailuresCauses, Avoidance, and Throubleshooting", Proceedings of $27^{\text {th }}$ Turbomachinery Symposium (College Station, TX: Texas A\&M University System Turbomachinery Laboratory, 2012), 129-180.

4. S. Ghosh and M. Callas. "Multi-Time Scaling Image Based Crystal Plasticity FE Models Dwell Fatigue Initiation in Polycrystalline Ti Alloys", Proceedings of the $1^{\text {st }}$ World Congress in Integrated Computational Materials Engineering (ICME), eds. J. Allison, P. Collins and G. Spanos (Hoboken, NJ: TMS, John Wiley \& Sons, 2011), 113-120.

5. M.F. Horstmeyer. "Computational Materials Engineering (ICME) for Metals: Using Multiscale Modelling to Invigorate Engineering Design with Science," (Hoboken, NJ: TMS, John Wiley \& Sons, 2012).

6. S.T. Wlodek, M. Kelly and D.A. Alden. "The Structure of René 88DT," Superalloys 1996, eds. R.D. Kissinger et al. (Warrendale, PA: The Minerals, Metals \& Materials Society, 1996), 129-136.

7. T. Straub et al. "Small-Scale Multiaxial Setup for Damage Detection into the Very High Cycle Fatigue Regime," Experimental Mechanics, 55(7) (2015), 1285-1299.

8. J. Miao, T.M. Pollock and J.W. Jones. "Microstructural Extremes and the Transition from Fatigue Crack Initiation to Small Crack Growth in a Polycrystalline Nickel-Base Superalloy," Acta Materialia, 60 (2012), 2840-2854.

9. J. Miao, T.M. Pollock and J.W. Jones. "Crystallographic Fatigue Crack Initiation in Nickel-Base Superalloy René 88DT at Elevated Temperature," Acta Materialia, 57 (2009), 5964-5974.

10. M. Seita et al. "The Dual Role of Coherent Twin Boundaries in Hydrogen Embrittlement", Nature Communications, 6 (2015), $1-6$.

11. H. Mughrabi et al. "Fatigue Crack Initiation by Cyclic Slip Irreversibilities in High-Cycle Fatigue", Fatigue Mechanisms: Advances in Quantitative Measurement of Physical Damage, eds. J. Lankford et al. (Philadelphia, PA: ASTM STP 811, ASTM, 1983), 5-45.

12. J.C. Stinville et al. "High Resolution Mapping of Strain Localization Near Twin Boundaries in a Nickel-Based Superalloy," Acta Materialia, 98 (2015), 29-42. 
13. J.C. Stinville et al. "A Combined Grain Scale Elastic-Plastic Criterion for Identification of Fatigue Crack Initiation Sites in a Twin Containing Polycrystalline Nickel-Base Superalloy," Acta Materialia, 103 (2016), 461-473.

14. A. Heinz and P. Neumann. "Crack Initiation During High Cycle Fatigue of an Austenitic Steel," Acta Metallurgica et Materialia, 38(10) (1990), 1933-1940. 